\title{
Methodology for user and user's life centered clinical evaluation of assistive technology (ULCEAT): Evaluation with prototype Roboticbed $^{\circledR}$
}

\author{
Mio Nakamura ${ }^{\mathrm{a}, \mathrm{c}, *}$, Miwa Sakiyama ${ }^{\mathrm{a}}$, Jun Suzurikawa ${ }^{\mathrm{a}}$, Shohei Tsukada ${ }^{\mathrm{b}}$, Akihiro Ohta $^{\mathrm{b}}$, \\ Yohei Kume $^{\mathrm{b}}$, Hideo Kawakami ${ }^{\mathrm{b}}$, Kaoru Inoue ${ }^{\mathrm{c}}$ and Takenobu Inoue ${ }^{\mathrm{a}}$ \\ ${ }^{a}$ Research Institute of National Rehabilitation Center for Persons with Disabilities, Tokorozawa, Japan \\ ${ }^{\mathrm{b}}$ Hospital Robot Strategic Business Unit, Panasonic Healthcare Co. Ltd., Kadoma, Japan \\ ${ }^{\mathrm{c}}$ Department of Occupational Therapy, Faculty of Health Sciences, Tokyo Metropolitan University, Tokyo, Japan
}

\begin{abstract}
Background: Clinical evaluation of novel assistive technologies is of utmost importance, as this would help in making such technologies practical and ensuring that they suit the user's requirements.

Objective: The purpose of this study is to develop a new methodology for the addition of user and user's life centered clinical evaluation of assistive technologies (ULCEAT) to conventional evaluation methods. The proposed ULCEAT methodology consists of two steps: evaluation by rehabilitation professionals and evaluation by potential users. These evaluations were performed to identify target users and to ensure the effective use of new assistive technologies (ATs); the evaluations were based primarily on qualitative research involving the rehabilitation professionals and the potential users.

Methods: The methodology was demonstrated by using it for the clinical evaluation of Roboticbed ${ }^{\circledR}$, which was developed by the Panasonic Corporation.

Results: The effective use of Roboticbed ${ }^{\circledR}$ was confirmed on the basis of the results, and three target user types were identified. The validity of this methodology was further confirmed by a user evaluation of Roboticbed ${ }^{\circledR}$ in an experimental environment. Conclusions: The findings of this study will be used to help conduct conventional clinical evaluations for novel and prototype ATs.
\end{abstract}

Keywords: Rehabilitation professionals, development of assistive technology, clinical evaluation, robot technology, qualitative research, user identification

\section{Introduction}

Assistive technologies (ATs) have been developed and put into practical use in order to support elderly and disabled individuals to lead a better life. The evaluation of such technologies is a critical component of the AT research and development process that links engineers and clinicians to individuals with disabilities [1].

${ }^{*}$ Corresponding author: Mio Nakamura, OTR, MS, Department of Assistive Technology, Research Institute, NRCD, Namiki 4-1, Tokorozawa, Saitama 359-8555, Japan. E-mail: nakamura-mio@rehab. go.jp.
Many methodologies for evaluating existing and commercialized ATs are known to exist, including six conceptual clinical evaluation models [2]: the $\mathrm{Hu}-$ man Activity-Assistive Technology (HAAT) model [3]; the International Classification of Functioning, Disability, and Health (ICF) model [4,5]; the Matching Personal and Technology (MPT) model [6]; Gitlin's biopsychosocial model of AT users' "careers" [7]; various social cognition decision-making theories [8]; and the Perceived Attributes Theory for predicting consumer adoption of product innovations [9]. The tools for psychological testing AT include the Quebec User Evaluation of Satisfaction with Assistive Technology (QUEST) [10] and the Psychosocial Impact of 
Assistive Devices Scale (PIADS) [11]. Furthermore, many evaluations focusing on wheelchair use in particular exist, including: Functional Evaluation in a wheelchair (FEW) [12]; the Life-Space Assessment Questionnaire (LSA-F) [13]; the Wheelchair Outcome Measure (WhOM) [14]; the Wheelchair Skills Program (WSP) [15]; the Power mobility Indoor Driving Assessment (PIDA) [16]; the Wheelchair Seating Discomfort Assessment Tool (WcS-DAT) [17]; and the Seating Identification Tool (SIT) [18]. However, not much evidence is available regarding clinical evaluation of prototypes and newly invented ATs.

According to that information which is available, clinical evaluation of prototype ATs is mostly carried out by specific organizations and by developers of new ATs [19-21]. For these evaluations, differing environments, assumptions, and batteries of evaluation have been used. For instance, Nihei et al. have proposed an evaluation protocol of failsafe wheelchair brakes for users with memory loss [22], and Inoue et al. presented a protocol for users with dementia [23] based on an evaluation protocol for cognitive AT [24]. These protocols are for devices with clear target users and use environments. However, no process exists for evaluating novel ATs under unclear effectiveness-of-use conditions. It is thus imperative to develop a prior evaluation methodology specific to each technology. Hammel et al. have proposed a comprehensive evaluation method for a rehabilitation robot system [1]. Their method has three phases: conceptual brainstorming, clinical feasibility testing, and viability testing. This method was applied within a human performance model to evaluate the impact of technological interventions on individuals with disabilities and the people with whom they interact (i.e., family, attendants, and rehabilitation professionals) within both personal contexts (i.e., home, hospital, school, work, and the community) and the context of the broader society. However, this method defines how a new AT can be created from a concept. Hence, it cannot be applied to a prototype AT. Jensen et al. discussed a project to involve users and professionals in the development of ATs by finding and systematizing new pathways for user-driven innovation (UDI) [25]. Although the UDI method is effective in identifying user requirements and problems, the target users must be determined by the developer.

Therefore, a prior evaluation involving the identification of both target users and effectiveness-of-use environments is required.

In this paper, we propose a methodology for a user and user's life centered clinical evaluation of AT (UL-
CEAT). We discuss our methodology (i.e., evaluation by rehabilitation professionals and users) and demonstrate it by using it to clinically evaluate Roboticbed ${ }^{\circledR}$, which was developed by the Panasonic Corporation.

\section{Development of ULCEAT methodology}

\subsection{Evaluation process}

The ULCEAT process consists of two steps, as shown in Fig. 1. The methodology, which describes the evaluations to be conducted before conventional clinical evaluations, is as follows:

Step 1: Evaluation by rehabilitation professional Purpose: To identify target users and effectiveness of use

Output: Identification of problems and user requirements

Step 2: Evaluation by potential users

Purpose: To establish the use environment Output: Identification of problems and user requirements

\subsection{Proposed ULCEAT methodology}

The ULCEAT methodology proposed here uses qualitative research methods. Such methods can be employed to uncover important variables and factors that can then be verified by objective testing [26]. Qualitative methods are suitable for identifying users, their needs, and effectiveness uses. Our methodology is as follows:

Step 1: Evaluation by rehabilitation professionals

1) Objective: To identify target users and effective uses of the technology

2) Study design: Qualitative research related to ethnography

3) Participants: Rehabilitation professionals We make clinical evaluation by rehabilitation professionals the first step because such professionals can help in identifying target users for ATs. On the basis of their expert knowledge and clinical experience with patients having various disabilities, they can provide insight into effective uses for ATs.

For Step 1, we recommend using a minimum of five participants in order to ensure observation of the most possible usability problems $[27,28]$. 


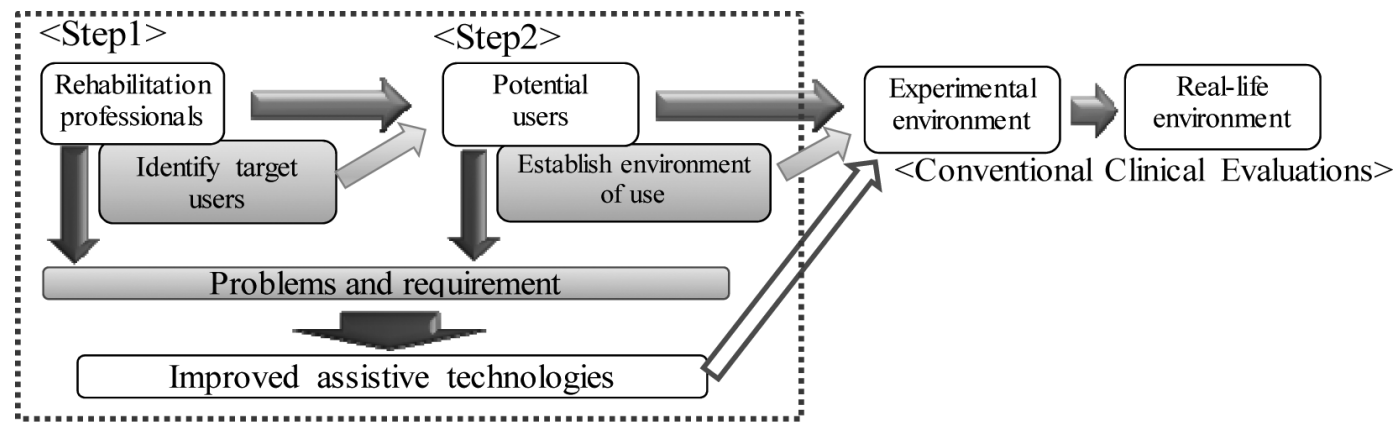

Fig. 1. UCEAT process.

4) Method: The experimental method involves (1) behavioral observation, and (2) an interview. In the behavioral observation, the use of Roboticbed ${ }^{\circledR}$ is observed in a simple setting, and the experimental processes are recorded with a video camera. An interview is then conducted using a semi-structured interview guide. Interview questions concern the participants' impressions of the trial run and their opinions of who would make suitable users of the AT [29].

\section{Step 2: Evaluation by potential users}

1) Objective: To establish the use environment

2) Study design: Ethnography-related qualitative research

3) Participants: Potential users picked on the basis of Step 1 results

For Step 2, we recommend using a minimum of five participants to ensure observation of the most possible usability problems $[27,28]$.

4) Method: The experimental method involves (1) behavioral observation, and (2) an interview. For behavioral observation, use of Roboticbed ${ }^{\circledR}$ is observed in a simple setting, and the experimental processes are recorded with a video camera. An interview is then conducted using a semistructured interview guide. Interview questions concern the participants' impressions of the trial run and the effectiveness of use of Roboticbed ${ }^{\circledR}$, as well the effectiveness of its means of use and the usefulness of the environment [29].

\section{Case study}

\subsection{Case: Use of the Roboticbed ${ }^{\circledR}$ for transfer and mobility}

As shown in Fig. 2, Roboticbed ${ }^{\circledR}$ helps transfer disabled people from bed to a wheelchair and facilitates general indoor mobility [30]. The device helps disabled people to carry out day-to-day activities with ease and improves quality of life. However, since Roboticbed ${ }^{\circledR}$ is a new device, no established criteria exist for its clinical evaluation; furthermore, it remains difficult to identify target users, set evaluation criteria, determine the risks involved, and plan for the effective use of the technology. Hence, this case study focuses on identifying these factors for the Roboticbed ${ }^{\circledR}$.

\subsection{Step 1: Evaluation by rehabilitation professionals}

\subsubsection{Method and analysis}

The participants in this step were five rehabilitation professionals: two occupational therapists, two physical therapists, and one nurse. Three were male and two female, and participants were aged between 20 and 60 . Their overall clinical experience was with physically disabled adults and elderly people in hospitals and within the community, and the lengths of their individual professional experience ranged from 5 to 28 years. The experiment in Step 1 involved two stages: observation and interview. The observation step was performed in the experimental space shown in Fig. 3, and participants' actions were recorded by two video cameras. Participants were instructed to perform the following six tasks:

1) Lie down on the bed

2) Shift from the bed to the wheelchair

3) Drive around the table

4) Go to the table and drink water from a cup placed there

5) Return to the initial position

6) Shift from the wheelchair to the bed

Interviews based on a semi-structured interview guide were conducted. Questions were asked concern- 
Table 1

Potential users for Roboticbed ${ }^{\circledR}$

\begin{tabular}{|c|c|c|}
\hline Users & Place & Advantages \\
\hline \multirow{5}{*}{$\begin{array}{l}\text { Individuals who wish } \\
\text { to promote autonomy } \\
\text { Individuals who often move } \\
\text { from bed to wheelchair }\end{array}$} & \multirow{5}{*}{$\begin{array}{l}\text { Home, nursing home, } \\
\text { indoors, outdoors } \\
\text { Home, nursing home, } \\
\text { indoors }\end{array}$} & No assistance required for transfer \\
\hline & & Allows user to move at will \\
\hline & & $\begin{array}{l}\text { Allows frequent shifting from bed to wheelchair } \\
\text { with minimum assistance }\end{array}$ \\
\hline & & Easy indoor mobility \\
\hline & & $\begin{array}{l}\text { User can choose activity (e.g., watch TV with } \\
\text { family, work on computer placed on table) }\end{array}$ \\
\hline \multirow{2}{*}{$\begin{array}{l}\text { Individuals who require } \\
\text { functional recovery training }\end{array}$} & \multirow{2}{*}{$\begin{array}{l}\text { Home, nursing home, } \\
\text { indoors }\end{array}$} & Makes moving out of bed easy \\
\hline & & Prevents disuse syndrome \\
\hline
\end{tabular}
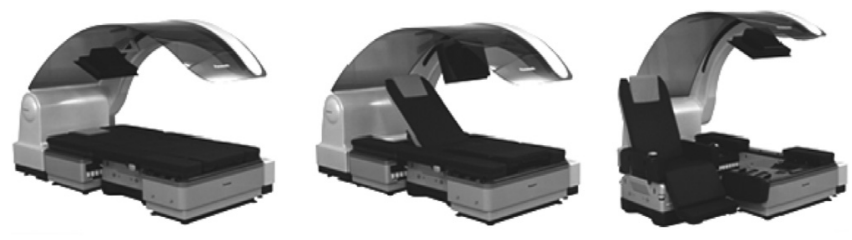

Bed

Recliner

$\Rightarrow \Rightarrow$ Wheelchair

Fig. 2. Roboticbed ${ }^{\circledR}$ positions.

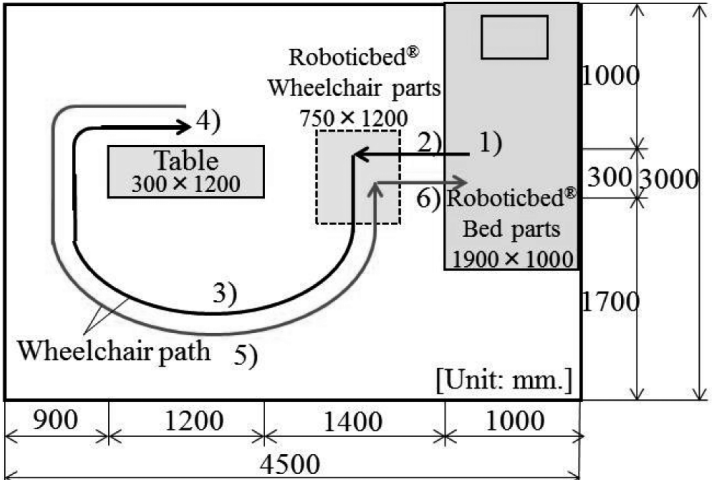

1) Lie down on the bed

2) Shift from the bed to the wheelchair

3) Drive around the table

4) Go to the table and drink water from a cup placed there

5) Return to the initial position

6) Shift from the wheelchair to the bed

Fig. 3. Experimental setup of Step 1 and Step 2.

ing participants' impressions of the test and their opinions as to who might be suitable target users.

Analysis of the interview results was based upon the KJ method [31,32], which was developed by cultural anthropologist Jiro Kawakita as a data gathering technique. In the KJ method, data are recorded regarding a group's descriptions of a selected item. This method is useful in the context of cooperative work, and is believed to facilitate "creativity development." The KJ method was used by the two occupational therapists involved in this study.

\subsubsection{Results of step 1}

\subsubsection{Potential users}

The experiment revealed three potential user types, as listed in Table 1: individuals hoping to enhance their autonomy, individuals who often need to move from their bed to a wheelchair, and individuals requiring functional recovery training. Table 1 also provides information about the environments in which each user type could use the bed and the advantages incurred for each.

\subsubsection{Specific requirements of Roboticbed ${ }^{\circledR}$}

Table 2 shows the specific requirements for Roboticbed ${ }^{\circledR}$ - classified as high-, medium-, and lowlevel - with many of these important in ensuring user safety. A few of these requirements can be modified on the basis of expert knowledge and clinical experience to meet user-specific needs.

\subsubsection{Expected effects of Roboticbed ${ }^{\circledR}$}

The results of the interview in which professionals helped identify target users for Roboticbed ${ }^{\circledR}$ based on their experience were quite unexpected. These participants saw the bed as being potentially useful in two ways: (1) in changing user attitudes and lessening physical and psychosocial burdens on caregivers, and (2) in providing users encouragement by facilitating independent mobility and thus a sense of freedom. Therefore, it can be concluded that rehabilitation professionals view Roboticbed ${ }^{\circledR}$ as a means for motivating people with physical disabilities to be independent. 
Table 2

Specific requirements for Roboticbed ${ }^{\circledR}$

\begin{tabular}{|c|c|c|c|}
\hline \multirow[t]{2}{*}{ Category } & \multicolumn{3}{|c|}{ Specific requirements } \\
\hline & High & Medium & Low \\
\hline Wheelchair & $\begin{array}{l}\text { - Give support to the user and help him/her } \\
\text { to maintain upright posture, (i.e., maintain } \\
\text { the head, trunk, bony pelvis, and inferior } \\
\text { limb in the most comfortable position) }\end{array}$ & $\begin{array}{l}\text { - Rearview system } \\
\text { - Change wheelchair size }\end{array}$ & - Damping during motion \\
\hline Bed & $\begin{array}{l}\text { - Identification of correct sleeping position } \\
\text { - Pressure sore prevention } \\
\text { - Waterproof }\end{array}$ & $\begin{array}{l}\text { - Create a space under the bed } \\
\text { - Functions to facilitate elevation and lower- } \\
\text { ing of the bed } \\
\text { - Adjustment of bed size to suit individual } \\
\text { users }\end{array}$ & $\begin{array}{l}\text { - Fixing bed rails } \\
\text { - Low-impact setting of med- } \\
\quad \text { ical device }\end{array}$ \\
\hline $\begin{array}{l}\text { Automatic } \\
\text { Posture } \\
\text { Change }\end{array}$ & $\begin{array}{l}\text { - Prevent the user from slipping off the seat } \\
\text { while reclining and tilting Roboticbed }{ }^{\circledR}\end{array}$ & $\begin{array}{l}\text { - Low-burden or stress-free posture during au- } \\
\text { tomatic operation }\end{array}$ & $\begin{array}{l}\text { - Seat width adjustability } \\
\text { - Adjusting the seat to suit } \\
\text { each user's inferior limb } \\
\text { condition }\end{array}$ \\
\hline $\begin{array}{l}\text { Input } \\
\text { Operation }\end{array}$ & - Easy handling of the remote control device & $\begin{array}{l}\text { - Merge switches } \\
\text { - Change size and configuration of the opera- } \\
\text { tion device }\end{array}$ & $\begin{array}{l}\text { - Remote control device for } \\
\text { caregiver } \\
\text { - Individualize input devices }\end{array}$ \\
\hline
\end{tabular}

Table 3

Participant profiles for Step 2

\begin{tabular}{|c|c|c|c|c|c|}
\hline ID & 1 & 2 & 3 & 4 & 5 \\
\hline Gender & $\mathrm{M}$ & $\mathrm{M}$ & $\mathrm{M}$ & $\mathrm{M}$ & $\mathrm{M}$ \\
\hline Age & 58 & 45 & 61 & 19 & 72 \\
\hline \multirow[t]{2}{*}{ Disease } & Parkinsonian & Cervical & Cervical & Muscular & Parkinsonian \\
\hline & Syndrome & Cord Injury & Cord Injury & Dystrophy & Syndrome \\
\hline FIM $^{*}$ & 68 & 25 & 25 & 31 & 50 \\
\hline Wheelchair Types & Not Used & Powered & Powered & Powered/Manual & Powered (Add-on power unit) \\
\hline Wheelchair usage & Not Used & Indoors/Outdoors & Indoors/Outdoors & Indoors/Outdoors & Indoors/Outdoors \\
\hline Bed usage & Used & Used & Used & Not Used & Used \\
\hline Lifter usage & Not Used & Used & Used & Not Used & Not Used \\
\hline Living Place & Nursing home & Home & Home & Home & Nursing home \\
\hline
\end{tabular}

*FIM = Functional Independence Measure, Motor score.

\subsection{Step 2: Evaluation by potential users}

\subsubsection{Method and analysis}

The participants in this step, whose profiles are summarized in Table 3, were five potential AT users. We extracted two categories of participants who meet the experimental conditions on the basis of the results of Step 1: individuals hoping to enhance their autonomy and those who often need to move from their bed to a wheelchair. These participants were asked questions in a semi-structured interview concerning their impressions of the trial run of Roboticbed ${ }^{\circledR}$ in relation to their perception of its use environment and the effective means for its use. The remainder of the methodology followed as described in Step 1.

\subsubsection{Results of step 2}

3.3.2.1. Task performance of Roboticbed ${ }^{\circledR}$ used by Potential Users
Table 4 shows the results of Step 2, in which it was determined whether participants were able to carry out tasks independently or with support.

\subsubsection{Use environment of Roboticbed ${ }^{\circledR}$}

Table 5 shows results pertaining to the use environment (which, participants indicated, was an indoor barrier-free environment) and the means of use for Roboticbed ${ }^{\circledR}$. In the experimental setup, there were five rooms: a bedroom, a living room, a dining room, a bathroom, and a washroom. Three common means of the use of Roboticbed ${ }^{\circledR}$ emerged. In the bedroom, the typical use was transferring, in which the participants moved from bed to wheelchair independently or with some support. In the living and dining rooms, the main reason for use was to perform activities alone or with family members, changing posture while being seated. In the washroom and the bathroom, the main reason for use was to move independently from the entrance. 
Table 4

Task performance conditions for Step 2

\begin{tabular}{llllllll}
\hline ID & & 1 & 2 & 3 & 4 & 5 & 6 \\
\hline Tasks & 1. Lie down on the bed & $\bigcirc$ & $\Delta$ & $\Delta$ & $\Delta$ & $\Delta$ & $\bigcirc$ \\
& 2. Transfer (Bed-W/C) & $\bigcirc$ & $\Delta$ & $\Delta$ & $\Delta$ & $\Delta$ & $\Delta$ \\
& 3. Drive around the table & $\bigcirc$ & $\bigcirc$ & $\bigcirc$ & $\bigcirc$ & $\bigcirc$ & $\bigcirc$ \\
& 4. Drink water from a cup placed on the table & $\bigcirc$ & $\bigcirc$ & $\bigcirc$ & $\bigcirc$ & $\bigcirc$ & $\bigcirc$ \\
& 5. Return to the initial position & $\Delta$ & $\Delta$ & $\bigcirc$ & $\bigcirc$ & $\bigcirc$ & $\bigcirc$ \\
6. Transfer (W/C-Bed) & $\bigcirc$ & $\Delta$ & $\Delta$ & $\Delta$ & $\Delta$ & $\bigcirc$ \\
\hline$=$ possible independently $\Delta=$ possible with support $\times$ = impossible.
\end{tabular}

$\bigcirc=$ possible independently $\Delta=$ possible with support $\times=$ impossible.

Table 5

Use environments and means of use for Roboticbed ${ }^{\circledR}$

\begin{tabular}{lll}
\hline Place & Environment & Examples \\
\hline Indoors & Bedroom & - Transferring between bed and W/C independently or with few supports \\
(Barrier-free Environment) & Living room/Dining room & - Reading books and operating PCs on the desk \\
& & - Watching TV and eating with family \\
& & - Changing posture while sitting \\
& & - Relaxing in the living room
\end{tabular}

Table 6

Participant profiles in evaluation with users in experimental environment

\begin{tabular}{|c|c|c|c|c|c|c|}
\hline ID & 1 & 2 & 3 & 4 & 5 & 6 \\
\hline Gender & $\mathrm{F}$ & $\bar{M}$ & $\mathrm{M}$ & $\bar{M}$ & $\bar{M}$ & $\mathrm{M}$ \\
\hline Age & 61 & 20 & 63 & 73 & 62 & 45 \\
\hline Disease & $\begin{array}{l}\text { Rheumatoid } \\
\text { Arthritis }\end{array}$ & $\begin{array}{l}\text { Muscular } \\
\text { Dystrophy }\end{array}$ & $\begin{array}{l}\text { Inherited } \\
\text { Neuropathy }\end{array}$ & $\begin{array}{l}\text { Parkinsonian } \\
\text { Syndrome }\end{array}$ & $\begin{array}{l}\text { Cervical } \\
\text { Cord Injury }\end{array}$ & $\begin{array}{l}\text { Cervical } \\
\text { Cord Injury }\end{array}$ \\
\hline FIM* & 47 & 31 & 30 & 50 & 25 & 25 \\
\hline Wheelchair Types & $\begin{array}{l}\text { Powered } \\
\text { (Add-on power unit) }\end{array}$ & $\begin{array}{l}\text { Powered/ } \\
\text { Manual }\end{array}$ & Powered & $\begin{array}{l}\text { Powered } \\
\text { (Add-on power unit) }\end{array}$ & Powered & Powered \\
\hline Wheelchair usage & $\begin{array}{l}\text { Indoors/ } \\
\text { Outdoors }\end{array}$ & $\begin{array}{l}\text { Indoors/ } \\
\text { Outdoors }\end{array}$ & $\begin{array}{l}\text { Indoors/ } \\
\text { Outdoors }\end{array}$ & $\begin{array}{l}\text { Indoors/ } \\
\text { Outdoors }\end{array}$ & $\begin{array}{l}\text { Indoors/ } \\
\text { Outdoors }\end{array}$ & $\begin{array}{l}\text { Indoors/ } \\
\text { Outdoors }\end{array}$ \\
\hline Bed usage & Used & Not Used & Used & Used & Used & Used \\
\hline Lifter usage & Not Used & Not Used & Used & Not Used & Used & Used \\
\hline Living Place & Home & Home & Home & Nursing home & Home & Home \\
\hline
\end{tabular}

${ }^{*}$ FIM $=$ Functional Independence Measure, Motor score.

\section{Validating the experimental methodology}

In order to validate the ULCEAT methodology discussed above, we formulated the following made two hypotheses,

1) Roboticbed ${ }^{\circledR}$ can be used by the potential users, who were identified in the Step1 evaluation, in the environment established in Step 2.

2) Different types of potential users showed different results.

In order to prove these hypotheses, we conducted evaluations with the users identified in Step1 by using the experimental environment from Step 2. In detail this experiments, the evaluation participants were se- lected from among the users in Step 1. In order to ensure safety in the experimental environment, we chose users from two out of the three identified user types: individuals hoping to increase their autonomy, and individuals who often move from bed to wheelchair. The experimental environment was the same indoor environment used in Step 2. The tasks tested were transferring between bed and wheelchair, performing activities, and moving between rooms with the wheelchair.

\subsection{User evaluation of the experimental environment}

\subsubsection{Method and analysis}

The participants were six people with disabilities; their profiles are shown in Table 6. The experiment con- 
Table 7

Task performance using Roboticbed ${ }^{\circledR}$

\begin{tabular}{llllllll}
\hline ID & 1 & 2 & 3 & 4 & 5 & 6 \\
\hline Tasks & 1. Lie down on the bed & $\Delta$ & $\Delta$ & $\Delta$ & $\Delta$ & $\Delta$ & $\Delta$ \\
& & $\Delta$ & $\Delta$ & $\Delta$ & $\Delta$ & $\Delta$ & $\Delta$ \\
& 2. Move from the bed to the wheelchair & $\Delta$ & $\bigcirc$ & $\bigcirc$ & $\bigcirc$ & $\bigcirc$ & $\Delta$ \\
3. Move to the work table & $\Delta$ & $\Delta$ & $\Delta$ & $\bigcirc$ & $\Delta$ & $\Delta$ \\
4. Perform activity at the table & $\Delta$ & $\bigcirc$ & $\Delta$ & $\bigcirc$ & $\Delta$ & $\bigcirc$ \\
5. Go out of the bedroom & $\Delta$ & $\bigcirc$ & $\bigcirc$ & $\Delta$ & $\bigcirc$ & $\bigcirc$ \\
6. Move to the corridor & $\Delta$ & $\bigcirc$ & $\Delta$ & $\Delta$ & $\bigcirc$ & $\bigcirc$ \\
7. Enter the living room and close the door & $\Delta$ & $\Delta$ & $\Delta$ & $\bigcirc$ & $\bigcirc$ & $\Delta$ \\
8. Move to the living room table, staying clear of the chair & $\Delta$ & $\Delta$ & $\Delta$ & $\Delta$ & $\bigcirc$ & $\bigcirc$ & $\Delta$ \\
9. Perform activities (i.e., drink water, watch TV) & $\Delta$ & $\bigcirc$ & $\Delta$ & $\Delta$ & $\bigcirc$ & $\bigcirc$ \\
10. Move to the corridor & $\Delta$ & $\bigcirc$ & $\Delta$ & $\Delta$ & $\bigcirc$ & $\bigcirc$ \\
11. Open the door & $\bigcirc$ & $\bigcirc$ & $\bigcirc$ & $\Delta$ & $\bigcirc$ & $\Delta$ \\
12. Return to the corridor & $\Delta$ & $\bigcirc$ & $\bigcirc$ & $\Delta$ & $\bigcirc$ & $\bigcirc$ \\
13. Enter the bedroom and return to the initial position & $\Delta$ & $\Delta$ & $\Delta$ & $\Delta$ & $\Delta$ & $\Delta$ \\
14. Move from the wheelchair to the bed & $\Delta$ & $\Delta$ & $\Delta$ & $\Delta$ & $\Delta$ \\
15. Lie down on the bed & $\Delta$ & $\Delta$
\end{tabular}

$\bigcirc=$ possible independently $\Delta=$ possible with support $\times=$ impossible.

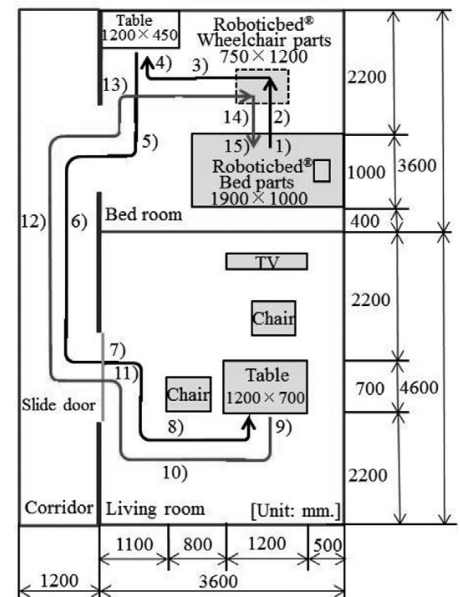

1) Lie down on the bed

2) Move from the bed to the wheelchair

3) Move to the worktable

4) Perform an activity at the table

5) Go out the bedroom

6) Move to the corridor

7) Enter the living room and close the door

8) Move to the living room table, staying clear of the chair

9) Perform activities

(e.g., drink water or watch TV)

10) Move to the living room

11) Open the door

12) Return to the corridor

13) Enter the bedroom and return to the initial position

14) Move from the wheelchair to the bed

15) Lie down on the bed

Fig. 4. Experimental setup of evaluation with users in experimental environment.

sisted of two steps: observation of actions and delivery of a questionnaire. The observed action step was per- formed within the experimental setup shown in Fig. 4. Participant actions were recorded by video cameras. In specific assignments, participants were instructed to perform the following 15 tasks:

1) Lie down on the bed

2) Move from the bed to the wheelchair

3) Move to the worktable

4) Perform an activity at the table

5) Go to the bedroom

6) Move to the corridor

7) Enter the living room and close the door

8) Move to the living room table, staying clear of the chair

9) Perform activities (e.g., drink water or watch TV)

10) Move to the living room

11) Open the door

12) Return to the corridor

13) Enter the bedroom and return to the initial position

14) Move from the wheelchair to the bed

15) Lie down on the bed

QUEST and PIADS (both in Japanese) were used to evaluate questionnaire answers. Generated scores were used in aggregate analysis.

\subsubsection{Results of the evaluation by users in an experimental environment}

\subsubsection{Results of task performance using Roboticbed ${ }^{\circledR}$}

Table 7 summarizes the results of the evaluation in which it was determined whether participants were able to carry out tasks independently or with support. 


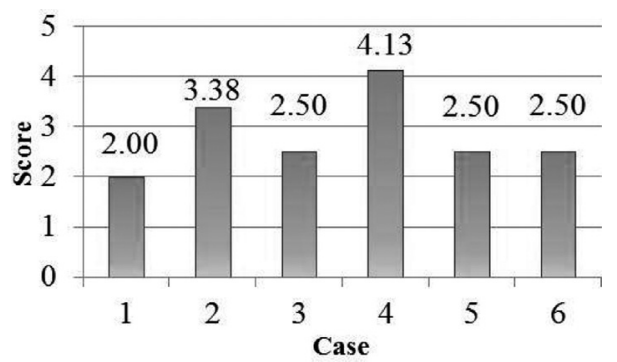

Fig. 5. QUEST score of Roboticbed ${ }^{\circledR}$.

\subsubsection{Results of QUEST and PIADS scoring}

The QUEST and PIADS results are shown in Figs 5 and 6 , respectively. The results are divided into two groups, with individuals hoping to increase their autonomy labeled as $\mathrm{C}, \mathrm{E}$, and $\mathrm{F}$, and individuals who often move from bed to wheelchair labeled as A, B, and D. The individuals who hoped to increase their autonomy showed lower QUEST and PIADS scores.

\section{Discussion}

\subsection{Methodology for prior clinical evaluation of assistive technology}

The proposed ULCEAT methodology consists of two steps: evaluation by rehabilitation professionals and evaluation by potential users. The results of Step 1 suggested three types of target users, and the results of Step 2 showed the effectiveness of use and the effective use environments of Roboticbed ${ }^{\circledR}$. These findings may be used to define target users and experimental conditions for conventional AT-evaluation methodologies, including user evaluation under experimental conditions and user evaluation in a real-life environment. Because such evaluations are usually carried out by specific organizations or by AT developers, differing evaluation environments, assumptions, and evaluation batteries had been previously used. In addition, some evaluations have not ensured suitability of conditions or users. To help define appropriate target users, uses by user type, and use environments, we combine Steps 1 and 2. From step 1, we can identify target users and appropriate uses, and from Step 2, we can establish use environment. The combination of these two steps provides an evaluator-independent tool and suitable setting for the evaluation of new ATs. We therefore believe that this ULCEAT methodology is effective for clinical evaluation.

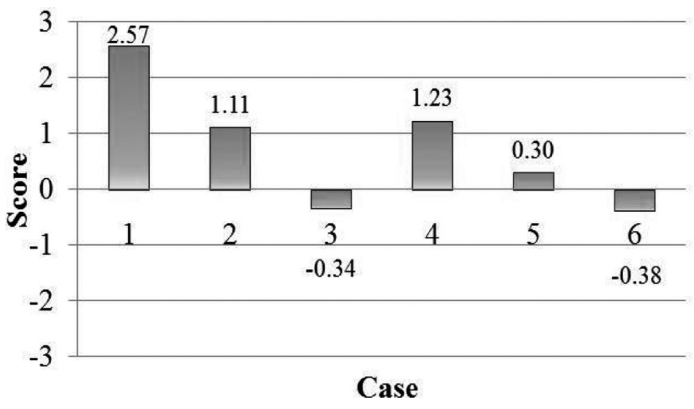

Fig. 6. PIADS score of Roboticbed ${ }^{\circledR}$.

\subsection{Validity of ULCEAT methodology}

The results of the experimental environment evaluation (Table 7) showed that all the participants were able to conduct each task either independently or with support. These results implied the verification of the first hypothesis about the validity of ULCEAT. The validity of the Step 1 evaluation was suggested from the perspective of the potential users. However, all participants needed support to perform the experimental tasks. This was thought to be because three were problems with matching the users' body functions and those of Roboticbed ${ }^{\circledR}$, as it was the first prototype. We pointed out the need for the modification from these results. As shown in Fig. 1, the results of Step 1 identified ways to improve Roboticbed ${ }^{\circledR}$. Therefore, we believed that these results also supported the validity of Step1. The validity of the Step 2 evaluation was suggested from the perspective of the use environment. As shown in Table 7, all the participants needed support for tasks 1, 2, 14 , and 15. However, at least one participant was able to accomplish the other tasks independently, which had an influence on the environment. These results would suggest that only the environmental factors played a role, without the users' factors. Therefore, we thought that it was implied for matching the experimental environment and Roboticbed ${ }^{\circledR}$. In addition, the environment settings realistically simulated a relatively large size house. These two facts supported the validity of Step 2 evaluation.

The QUEST and PIADS results shown in Figs 5 and 6 seem to verify the second hypothesis. Table 8 shows characteristics of the two types of potential users who participated in the evaluation. Participants $\mathrm{C}, \mathrm{E}$ and $\mathrm{F}$ belong to the upper category of individuals who hope to promote autonomy and want to use Roboticbed ${ }^{\circledR}$ indoors and outdoors. Meanwhile, participant A, B and D belong to the lower category of individuals who often move from their bed to a wheelchair and want to use 
Table 8

Relations of participant types to environment

\begin{tabular}{llcc}
\hline & & \multicolumn{2}{c}{ Environment } \\
\cline { 3 - 3 } Person & Individual who hopes to promote autonomy & Outdoor & Indoor \\
\cline { 2 - 3 } & Individual who often moves from bed to wheelchair & $\bigcirc$ & $\bigcirc$ \\
\hline
\end{tabular}

it indoors. The QUEST and PIADS scores for participants $\mathrm{C}, \mathrm{E}$ and $\mathrm{F}$ were relatively lower than those for participants A, B and D. This was consistent with this hypothesis with respect to outdoor versus indoor use requirements. Thus, we confirmed our hypothesis that users of different types showed different results.

From these discussions, the validation of our proposed ULCEAT was implied.

\subsection{Limitations}

In this study, the experiment of Step 2 could only be conducted with two categories of users out of the three categories of users found in the results of Step 1. The remaining category of users was composed of individuals who required functional recovery training in a hospital after orthopedic surgery. Thus, they were not able to come to the location where the experiment was conducted. These users have to be evaluated in a real-life environment without Step 2 and the evaluation in an experimental environment. In order to perform evaluations in a real-life environment, it is necessary to identify four important experimental conditions: whether or not it is possible to use Roboticbed ${ }^{\circledR}$, the most useful environment, the problems with its use, and whether it is safe. First, it is necessary to confirm these conditions based on the results from the two categories of users who were able to participate in the experiment. Furthermore, the results from Step 1 should be analyzed in detail to identify other conditions. In addition, the evaluation in a real-life environment must be carefully conducted. A trial use will be needed in advance. These are some of the future plans for our project.

Furthermore, the proposed ULCEAT methodology was confirmed through a clinical evaluation of Roboticbed $^{\circledR}$ in an experimental environment. However, we conducted our evaluation and validated the ULCEAT methodology using only Roboticbed ${ }^{\circledR}$. To better validate our methodology, it will be necessary to further test the ULCEAT methodology by evaluating more participants and verifying more novel or prototype ATs.

\section{Conclusions}

The purpose of this study was to develop the ULCEAT methodology for the clinical evaluation of novel and prototype ATs. The ULCEAT methodology we propose consists of two steps: evaluation by rehabilitation professionals and evaluation by potential users. Evaluation is mainly based on qualitative research assisted by rehabilitation professionals and users. Our proposed methodology was confirmed through clinical evaluation of Panasonic's Roboticbed ${ }^{\circledR}$. From our results, three types of users were identified, and their ability to effectively use Roboticbed ${ }^{\circledR}$ was confirmed. The results also demonstrated the utility of evaluation by rehabilitation professionals and potential users of the ULCEAT methodology. Therefore, we conclude that this evaluation method is implied for the conventional evaluation of ATs.

\section{Acknowledgements}

This research was partially supported by NEDO. We also thank the participants, their families, and their caregivers for their kind cooperation.

\section{References}

[1] J.M. Hammel, The role of assessment and evaluation in rehabilitation robotics research and development: Moving from concept to clinic to context. IEEE Transactions on Rehabilitation Engineering, 3(1): 56-61, 1995.

[2] J.A. Lenker and V.L. Paquet, A new conceptual model for assistive technology outcomes research and practice. Assitive Technology, 16(1): 1-10, 2004.

[3] A.M. Cook and S.M. Hussey, Assistive technologies principles and practice (2nd ed.), ST. Louis, MO: Mosby, 2002.

[4] J.A. Lenker and J.W. Jutai, Assistive technology research and practice: What role for ICF? Paper presented at the 8th North American Collaborating Center (NACC) conference on ICF, Toronto, ON, 2002.

[5] World Health Organization. ICF: International classification of functioning, disability, and health. Geneva: Author., 2001.

[6] M.J. Scheer, Matching person and technology, Webster, NY: Institute for Matching Person and Technology, 1998. 
[7] L.N. Gitlin, From hospital to home: Individual variations in experience with assistive devices among older adults, In D.B. Gray, L.A. Quantrano, M.L. Lieberman (Eds), Designing and using assistive technology, pp. 117-135, Baltimore, MD: Paul H. Brookes, 1998.

[8] I. Ajzen, Perceived behavioral control, self-efficacy, locus of control, and the theory of planned behavior, Journal of Applied Social Psychology, 32(32): 1-20, 2002.

[9] V.H. Carr, Technology adoption and diffusion, National Library of Medicine, The Collaboration for High Performance Computing and Communications, Retrieved November 11, 2003, from http://colab.nim.nih.gov/tutorialspublicationsan dmaterials/sourcebook/adoptiondiffusion.html.

[10] L. Demers, R. Weiss-Lambrou and B. Ska, Item analysis of quebec user evaluation of satisfaction with assistive technology (QUEST), Assistive Technology, 12(2): 96-105, 2000.

[11] T. Inoue et al., Standardization of J-PIADS (Psychosocial Impact of Assistive Devices Scale), Selected Papers from the Japanese Conference on the Advancement of Assistive and Rehabilitation Technology, IOS Press, pp. 49-54, 2011.

[12] L. Tamara, et al. Test-retest reliability and cross validation of the functioning everyday with a wheelchair instrument, Assistive Technology, 19(2): 61-77, 2007.

[13] C. Auger et al., Development of a French-Canadian version of the life-space assessment (LSA-F): content validity, reliability and applicability for power mobility device users, disability and rehabilitation, Assistive Technology, 4(1): 31-41, 2009.

[14] W.B. Mortenson et al., Measuring wheelchair intervention outcomes: Development of the wheelchair outcome, disability and rehabilitation, Assistive Technology, 2(5): 275-285, 2007.

[15] R.L. Kirby and R.A. Cooper, Applicability of the wheelchair skills program to the Indian context, disability and rehabilitation, 29(11-12): 969-972, 2007.

[16] D. Dawson, et al. Development of the power-mobility indoor driving assessment for residents of long-term care facility: a preliminary report, Canadian Journal of Occupational Therapy, 61(5): 269-76, 1994.

[17] B.A. Crane, Development of a consumer-driven wheelchair seating discomfort assessment tool (WcS-DAT), International Journal of Rehabilitation Research, 27(1): 85-90, 2004.

[18] W.C. Miller, et al. Development and preliminary assessment of the measurement properties of the seating identification tool (SIT), Clinical Rehabilitation, 18(3): 317- 325, 2004.

[19] H.Y. Liu, J. Pearlman, R. Cooper, E.K. Hong, H. Wang, B. Salatin and R.A. Cooper, Evaluation of aluminum ultralight rigid wheelchairs versus other ultralight wheelchairs using
ANSI/RESNA standards. J Rehabil Res Dev, 47(5): 441-55, 2010.

[20] A.J. Rentschler, R. Simpson, R.A. Cooper and M.L. Boninger, Clinical evaluation of Guido robotic walker. J Rehabil Res Dev, 45(9): 1281-93, 2008.

[21] J.J. Harrow, P. Malassigné, A.L. Nelson, R.P. Jensen, M. Amato and P.L. Palacios, Design and evaluation of a stand-up motorized prone cart. J Spinal Cord Med, 30(1): 50-61, 2007.

[22] M. Nihei, et al. Evaluation of failsafe wheelchair brake for users with memory loss in clinical use: Short-term evaluation of function, assistive technology from adapted equipment to inclusive environments, Assistive Technology Research, 25: 41-46, 2009.

[23] T. Inoue, et al. Development by a field-based method of a dailyplan indicator for people with dementia, Assistive Technology Research, 25: 364-373, 2009.

[24] L. Jensen, et al. How cognitive assistive technology can secure active living for people with dementia, Journal of Rehabilitation Research, 138: 15-18, 2009.

[25] L. Jensen, et al., User communities for innovation of assistive technology: Results from a demonstrations project about userdriven innovation, Everyday Technology for Independence and Care, IOS Press, pp. 987-994, 2011.

[26] A.D.V. Dabbs, B. Myers, K. McCurry, J. Dunbar-Jacob, R. Hawkins, A. Begey and M. Dew. User-centered design and interactive health technologies for patients, Computers Informatics Nursing, 27(3): 175-183, 2009. 110.1097/NCN.1090b $1013 \mathrm{e} 31817 \mathrm{c} 31817 \mathrm{c}$.

[27] J. Nielsen, Estimating the number of subjects needed for a thinking aloud test. Int J Hum-Comput Stud, 41(3): 385-397, 1994. (in Japanese).

[28] Y.H. Oda, Ethnography Beginning Guide. Shunjyu Company, 2010. (in Japanese).

[29] M. Kurosu, et al. User engineering beginning guide-thinks usability/objectivization approach in ISO13407. Kyouritushuppan Company, 1999. (in Japanese)

[30] Y. Kume, et al. Development of the Roboticbed ${ }^{\circledR}$, Journal of Automotive Technology, 64(5): 31-34, 2010. (in Japanese).

[31] J. Kawakita, Continuation of Methodology of the Creativity Techniques. Chukou-Shinsho, 1970. (in Japanese).

[32] Y. Htamura, Creation and Architectonics of Technology, pp. 255-301. Iwanami Bookstore, 2006. (in Japanese).

[33] M. Scherer, The impact of assistive technology on the lives of people with disabilities. In D.B. Gray, L.A. Quatrano and M.L. Lieberman, (Eds), Designing and Using Assistive Technology: The Human Perspective. Baltimore: Paul H. Books, 1998. 